\title{
Early-Onset Schizophrenia: A Special Phenotype of the Disease Characterized by Increased MTHFR Polymorphisms and Aggravating
}

\section{Symptoms}

Lin Wan

Jing Wei

Department of Psychological Medicine, Peking Union Medical College Hospital, Chinese Academy of Medical Sciences \& Peking Union Medical College, Beijing, People's Republic of China
Correspondence: Jing Wei

Department of Psychological Medicine, Peking Union Medical College Hospital, Chinese Academy of Medical Sciences \& Peking Union Medical College, Shuaifuyuan I, Dongcheng District, Beijing, People's Republic of China Tel +86-10-61956061

Email weijing@pumch.cn
Background: Patients with early-onset schizophrenia usually exhibit more severe symptoms, revealing a potentially distinctive disease phenotype. Methylenetetrahydrofolate reductase (MTHFR) is a critical enzyme in folate conversion and methylation modification associated with the disease. We aimed to investigate the potential effects of MTHFR polymorphisms and related methylation patterns in patients with early-onset schizophrenia, which implies special phenotypes of schizophrenia.

Methods: In 177 patients with schizophrenia, MTHFR polymorphism at three sites (C677T, A1298C, and G1793A) and the Positive and Negative Syndrome Scale (PANSS) were tested. Differential methylation positions (DMPs) and enrichment of genes and related pathways were analyzed by testing the genomic methylation level. Catechol-O-methyltransferase (COMT), solute carrier family 6 member 4 (SLC6A4), neuregulin1 (NRG1), and brainderived neurotrophic factor $(B D N F)$ were selected to evaluate the methylation levels of specific $\mathrm{CpG}$ regions by pyrosequencing.

Results: Higher levels of symptom severity and MTHFR polymorphisms and lower levels of global DNA methylation in patients with early-onset schizophrenia were observed in this study. SLC6A4 was hypermethylated, and BDNF was hypomethylated in specific regions of patients with early-onset schizophrenia.

Conclusion: Aggravating symptoms, increased MTHFR polymorphisms, and reduced genomic methylation levels may be characteristics and underlying mechanisms of early-onset schizophrenia, which implies a special disease phenotype. Beyond that, specific genes and biological pathways may imply the potential phenotype of schizophrenia.

Keywords: schizophrenia, phenotype, onset age, MTHFR, polymorphism, methylation

\section{Introduction}

Schizophrenia is one of the most severe psychiatric diseases characterized by a series of clinical syndromes, including abnormalities in cognition, thoughts, emotions, and behaviors. In 2013, schizophrenia was first classified by spectrum disorder in the Diagnostic and Statistical Manual of Mental Disorders, Fifth Edition (DSM-5), which consists of schizoid disorder, delusion, schizophrenia-like disorder, schizophrenia, and schizoaffective disorder. It is explicit for us to realize the complexity of schizophrenia spectrum disorders. In clinical practice, schizophrenia is the most investigated type, resulting in high disability and social burden. To 
better understand the pathogenesis of this disease and develop effective treatments, some of its phenotypes have been proposed as a category of symptoms, including paranoid, hebephrenia, catatonic, undifferentiated, and straightforward types. Another system divides schizophrenia into two types, types I and II, characterized by positive and negative symptoms. Generally, a related hypothesis supports the corresponding phenotypes of schizophrenia, such as dopaminergic divergence, in patients with type I and II schizophrenia. ${ }^{1}$

Schizophrenia usually attacks the youth stage, leading to significant retardation in growth and development, which causes a disability burden during the rest of a lifetime. It is critical to elucidate the molecular and clinical features of early-onset schizophrenia. Schizophrenia is often found to originate between ages of 10 and 30 years, while the periods of 10-20 and 2030 years are two of the onset peaks, respectively. ${ }^{2}$ It is generally revealed that subjects with schizophrenia with earlier onset age ( $<20$ years old) have more severe symptoms than patients with schizophrenia with other ages of onset. ${ }^{3}$ Retardant maturation has been observed in the brain regions of patients with early-onset schizophrenia, including the frontal and temporal lobes. ${ }^{4,5}$ Apart from clinical and histological analyses at the macroscopic level, molecular theories, including gene and related modifications, could be acquired with the special features of early-onset patients. For mature divergence in brain regions of patients with schizophrenia with different onset ages, neurodevelopmental disorders may exist in the pathogenesis of schizophrenia. ${ }^{6,7}$

As for human neurodevelopmental processes, folate is an essential substance that can prevent developmental disorders, such as neural tube defects. MTHFR is a critical enzyme that regulates folate conversion and metabolism, thereby contributing to the intracellular biotransformation of folate. The enzyme activity of MTHFR has been reported to be impaired by gene polymorphisms at specific sites. The animal models and clinical features of schizophrenia studies indicated that high MTHFR polymorphism might be associated with the elevation of disease risk and earlier onset age. ${ }^{8-11}$ However, some studies have concluded that MTHFR polymorphism does not affect schizophrenia risk or onset age. ${ }^{12,13}$ Although the conclusions between MTHFR and schizophrenia are inconsistent, the gene polymorphisms may contribute to the features of schizophrenia at different ages of onset. In addition to the alteration of MTHFR activity caused by gene polymorphisms, the methyl group supply downstream of folate conversion is affected, and the related methylation modification may be involved in the onset and clinical features of schizophrenia. ${ }^{14}$

Our study aimed to investigate the potential effects of MTHFR polymorphism and related methylation changes in patients with early-onset schizophrenia, which implies special phenotypes of schizophrenia (early-onset and adult-onset). This research provides a view of molecular variation in early-onset schizophrenia, which may contribute to clinical judgment and treatment as a potential special phenotype of the disorder. There has been little research on the molecular exploration of patients with schizophrenia with divergent onset ages. In this process, the severity of symptoms, MTHFR polymorphisms, and methylation levels of both genome and schizophreniarelated genes in patients with schizophrenia at different ages of onset were explored. The enrolled polymorphic sites included the MTHFR activity and function-correlated variants of C677T (rs1801133), A1298C (rs1801131), and G1793A (rs2274976). ${ }^{15-17}$ As the gene polymorphisms in the folate metabolism pathway have a combined function characterized by multi-site effects, ${ }^{18}$ we tested both singleand multi-site polymorphisms of MTHFR. MTHFR and folate effects may be executed through the methylation modification process, affecting the expression levels of functional genes. Enrolled patients with the TT genotype of C677T (decrease in enzyme activity by $70 \%$ ) $^{15,16}$ and high Positive and Negative Syndrome Scale (PANSS) scores were selected to determine methylation levels.

\section{Materials and Methods}

\section{Subjects}

All procedures complied with the latest version of the Declaration of Helsinki. The source of the patient samples was from Beijing Clinical Data and Sample Database of Mental Disorders. The ethical committee approved the study project of the hospital, and informed consent was obtained for experimentation with human subjects. Written informed consent was obtained from the parents of the enrolled patients $<18$ years of age in this study. 177 patients with schizophrenia (114 early-onset and 59 adultonset) were enrolled at an average age of 24 . The age of onset was defined as the first time that positive symptoms appeared, rather than negative symptoms or other nonpsychotic symptoms. No significant difference was found in the demographic data of sex between patients at 
Table I Demographic Characteristics of Enrolled Subjects

\begin{tabular}{|l|c|c|c|c|}
\hline Onset Age & Number $^{\dagger}$ & Mean Age $^{\ddagger}$ & Onset Age & Duration $^{\S}$ \\
\hline Early & $114(55 / 59)^{\top}$ & $16.1(16.6 / 15.8)$ & $13.6(13.4 / 13.7)$ & $30.5(32.7 / 28.4)$ \\
Adult & $63(36 / 27)$ & $42.3(41.2 / 43.4)$ & $32.6(32.1 / 33.4)$ & $130.0(130.1 / 128.7)$ \\
\hline
\end{tabular}

Notes: ${ }^{\dagger}$ No significant difference in sex frequency between patients with divergent onset ages; ${ }^{\ddagger}$ No significant difference in mean age between patients with divergent onset ages; ${ }^{\S}$ Count in months; ${ }^{~ I D a t a ~ i n ~ b r a c k e t s ~}(\mathrm{a} / \mathrm{b})$ represent male and female patients, respectively.

different ages of onset. The demographic characteristics of the enrolled subjects are shown in Table 1.

The enrolled patients were diagnosed according to the DSM-V criteria for schizophrenia based on two experienced physicians' judgment. The diagnostics was based on an interview of MINI Adult revised by TCH 2012-12-10 Information on demographics and PANSS scores were acquired. Two trained raters who were residents of psychiatry were involved in the assessment of PANSS. They were trained in a brief video-based training program. Participants performed a baseline PANSS rating based on a patient's video being interviewed using the simplified positive and negative symptoms interview (SNAPSI). Subsequently, all participants were trained in five successive, standardized weekly sessions. The results were analyzed with respect to the conventional criteria of concordance with standard expert ratings and interrater reliability. After two weekly sessions, approximately $75 \%$ of the participants rated the acceptable deviations of the gold standard. All enrolled subjects were of Han Chinese ethnicity and were in the stationary phase with no acute attack in the last six months. Exclusion criteria included: psychotic disorder due to another somatic disorder (nervous or non-nervous systems); combined diagnosis with other psychiatric disease besides schizophrenia.

The medication was an important factor for PANSS scores and DNA methylation of the genes. We collected the names and subject amounts of different antipsychotic regimens in early- and adult- onset groups (Supplemental Table 1). Then by calculating the mean doses of each drug weighted by sample size, we divided them by the weighted mean olanzapine dose to obtain olanzapine equivalents. Based on the comparison of mean equivalents dosage between early- and adult- onset groups, there is no statistical difference between them (Supplemental Table 2). As for other medication like Modified Electroconvulsive Therapy (MECT), it indicated that there was no statistical difference between the two groups (Supplemental Table 3).

Age is an important influencing factor on the concentration of folate and homocysteine. DNA methylation of genes is also different by age and antipsychotics. Then we performed analysis to adjust folate, homocysteine and methylation levels in age, and methylation level in antipsychotics, with consideration of early- and late- onset schizophrenia. While no significant correlation between antipsychotics or age and genomic methylation level of schizophrenic patients was found (Supplemental Table 4). No significant correlation was found between age and genomic methylation level of in schizophrenic patients of different onset age (Supplemental Table 5), and no significant difference was found in folate or Hcy levels between early- and adult-onset groups of schizophrenia (Supplemental Figure 1).

\section{Peripheral Samples}

Blood samples were collected from the psychiatric department of the hospital. Blood components were isolated to obtain the white blood cells and serum. DNA samples were extracted from the white blood cells for polymorphism and methylation assays. Other information was collected apart from PANSS ratings, including gender, age, onset age, duration of disease, family history, and Clinical Global Impression (CGI).

\section{MTHFR Polymorphism Assay}

Three polymorphic sites of MTHFR, including C677T (rs1801133), A1298C (1801131), and G1793A (rs2274976), were tested using a TaqMan fluorescence probe in the ABI PRISM 7500 Sequence Detection System (Applied Biosystems, Foster City, CA, USA). PCR reaction condition: total volume of $25 \mu \mathrm{L}$ system included Probe $0.63 \mu \mathrm{L}$, TaqMan Universal Master Mix II 12.5 $\mu \mathrm{L}$ (DNA Polymerase, Uracil-N glycosylase, dNTPs with dUTP, Passive Reference, Optimized buffer components), distilled water $6.87 \mu \mathrm{L}$, and $50 \mathrm{ng}$ genomic DNA. The samples were pre-degenerated at $95^{\circ} \mathrm{C}$ for 10 min, denatured for 35 cycles at $93^{\circ} \mathrm{C}$ for $40 \mathrm{~s}$, and finally annealed/extended at $60^{\circ} \mathrm{C}$ for $1 \mathrm{~min}$.

In addition to the single-site study of MTHFR polymorphisms, we also focused on the effects of multiple sites by calculating the total risk allele load, which may be 
a more comprehensive way to reflect the function of MTHFR. By summing the number of risk alleles at the three polymorphic sites, the total risk allele number was acquired to evaluate each subject. We compared the different frequencies of the overall risk allele numbers among different age groups of onset to determine the relationship between MTHFR polymorphisms and the different origins of schizophrenia and related symptoms. At the same time, we explored the interaction between the potential effects of methylation in MTHFR and the age of onset.

\section{Folate and Homocysteine (Hcy) Assay}

Folate levels were tested using the Elecsys Folate III Kit and Chemiluminescence apparatus (Cobas 8000-E602, Roche). In contrast, Hcy levels were examined using the Hcy Assay Kit (Leadmanbio) and Chemistry Analyzer (AU5800 Series, Beckman Coulter) according to the manufacturer's instructions.

\section{Genomic DNA Methylation Assay}

Sixteen schizophrenia patients with the TT genotype of MTHFR C677T and PANSS scores higher than 60 were chosen for genomic DNA methylation determination. Approximately $500 \mathrm{ng}$ of genomic DNA from each sample was used for sodium bisulfite conversion using the DNA Methylation Gold Kit (Zymo Research, USA) following the manufacturer's standard protocol. According to the manufacturer's instructions, genome-wide DNA methylation was assessed using the Illumina Infinium Human Methylation $850 \mathrm{~K}$ BeadChip (Illumina Inc., USA). The array data were analyzed using the ChAMP package in $\mathrm{R}$ software to determine the methylation level. The methylation status of all probes was denoted as the $\beta$ value, which is the ratio of the methylated probe intensity to the overall probe intensity. CpG sites with $|\Delta \beta| \geq 0.20$, between patients and controls, and an adjusted P-value $\leq$ 0.05 , were considered differentially-methylated sites. A $\mathrm{CpG}$ position was deemed to be hypermethylated if $\Delta \beta \geq 0.20$, or hypomethylated if $\Delta \beta \leq-0.20$. The average $\beta$ values of promoters and $\mathrm{CpG}$ islands were compared between the disease and normal groups. ${ }^{19}$

\section{Gene-Specific Methylation Assay}

For schizophrenia-related genes, including COMT, $S L C 6 A 4, N R G 1$, and $B D N F$, we analyzed six, four, seven, and six CpGs of their promoters. Through methylation modification, sulfite-modified DNA purification, methylation-specific PCR, and pyrosequencing assays, the DNA methylation levels at specific sites were quantified. Bisulfite treatment was conducted using the DNA Methylation Kit (Qiagen), and primers were designed by PSQ Assay Design (Qiagen). The tested sequences of these genes are listed below:

COMT: 5'-TGCCCGCCGCGCTGCCTGCGCCGGAC CGGGGCGGGTC-3';

SLC6A4: 5'-CCCCGACACACACACACGCTCGCAG GGAGGAGCGGAGCGCGGA-3';

NRG1: 5'-CGCTGGGTGGCCGCGGCGGCGACA GC-3';

BDNF: 5'-CCGG CCCGGCTGGGGAGCGGAGCGC GGTCTCGGC-3'.

\section{Statistical Analysis}

The data were processed using GraphPad Prism 6.0c, SPSS statistics 24 , and charts and diagrams were generated. The $\chi^{2}$ test was used to compare the differences in MTHFR polymorphism frequencies among patients with schizophrenia at different ages of onset. As there was column with less than five subjects, Fisher exact test was used to analyze. One-way analysis of variance (ANOVA) with post-hoc comparisons were used for symptoms and folate levels, as well as the Hcy analysis in patients at different ages of onset. Multiple linear regression was used to determine the multi-factor effects on the symptoms. In the methylation analysis of DMPs, empirical Bayes and linear regression were used to calculate the $\mathrm{P}$-value and adjusted P-value (FDR) after Benjamin and Hochberg's multiple tests. One-way ANOVA was used for genomic and gene-specific methylation analysis, whereas enrichment analysis was used for gene ontology (GO) analysis. This process also contained hypergeometric distribution and Fisher tests, while correlated terms were acquired from the GO database (http://www.geneontology.org). ${ }^{19}$

\section{Results and Statistical Analyses Analysis of Onset Age Prediction MTHFR Polymorphisms, Folate, and Hcy in Patients of Different Onset Ages}

MTHFR genotypes distributed in Hardy-Weinberg equilibrium for each group $(p>0.05)$ are shown in Table 2 . Linkage disequilibrium was observed between the C677T and A1298C polymorphisms $\left(\mathrm{R}^{2}=0.20, \mathrm{D}^{\prime}=1\right)$. Because of the non-random association of different loci, there may be interactions between C677T and A1298C in mutual allele or genotype frequency. We then focused on single and multiple 
Table 2 MTHFR Polymorphisms in Patients of Different Onset Ages

\begin{tabular}{|c|c|c|c|c|c|c|c|c|c|c|c|c|c|c|c|}
\hline \multirow[t]{2}{*}{ Sites } & \multicolumn{5}{|c|}{ C677T } & \multicolumn{5}{|c|}{ AI $298 C$} & \multicolumn{5}{|c|}{ GI793A } \\
\hline & CC & CT & TT & C & $\mathbf{T}$ & AA & $A C$ & CC & A & C & GG & GA & AA & G & $\mathbf{A}$ \\
\hline \multicolumn{16}{|l|}{ Early } \\
\hline Total & 25 & 52 & 37 & 102 & 126 & 79 & 32 & 3 & 190 & 38 & 98 & 16 & 0 & 212 & 16 \\
\hline HWE & \multicolumn{5}{|c|}{$0.4 I I$} & \multicolumn{5}{|c|}{0.910} & \multicolumn{5}{|c|}{0.422} \\
\hline \multicolumn{16}{|l|}{ Adult } \\
\hline Total & 15 & 29 & 19 & 59 & 67 & 52 & 10 & I & 114 & 12 & 58 & 5 & 0 & 121 & 5 \\
\hline HWE & \multicolumn{5}{|c|}{$0.48 I$} & \multicolumn{5}{|c|}{0.379} & \multicolumn{5}{|c|}{0.678} \\
\hline$\chi_{\text {Total }}^{2}$ & \multicolumn{2}{|c|}{0.133} & & \multicolumn{2}{|c|}{0.143} & \multicolumn{2}{|c|}{3.701} & & \multicolumn{2}{|c|}{3.414} & \multicolumn{2}{|c|}{ I.443 } & \multicolumn{3}{|c|}{1.352} \\
\hline$d f$ & \multicolumn{2}{|c|}{2} & & \multicolumn{2}{|c|}{ I } & \multicolumn{3}{|c|}{2} & \multicolumn{2}{|c|}{1} & \multicolumn{2}{|r|}{2} & \multicolumn{3}{|c|}{ I } \\
\hline $\mathrm{P}$ & \multicolumn{2}{|c|}{0.9358} & & \multicolumn{2}{|c|}{0.7055} & \multicolumn{2}{|c|}{0.1572} & & \multicolumn{2}{|c|}{0.0647} & \multicolumn{2}{|c|}{0.2296} & \multicolumn{3}{|c|}{0.2449} \\
\hline
\end{tabular}

Note: ${ }^{\dagger}$ Comparison in genotype and allele frequencies of three polymorphisms in patients of different onset ages.

Abbreviation: HWE, Hardy Weinberg Equilibrium.

sites of MTHFR polymorphisms in patients with schizophrenia. For C677T, A1298C, and G1793A, there was no significant difference in genotype or allele frequency among patients of different age groups. After gender stratification, neither male nor female patients with schizophrenia in these groups expressed a significantly different frequency of genotype or allele. The overall risk allele number of polymorphic sites was tested, including C677T, A1298C, and G1793A, to elucidate the total risk allele load of multiple sites of MTHFR polymorphisms. The total risk allele number of the multi-site polymorphism model ranged from 0 to 6 , while the number of enrolled subjects ranged from 0 to 3 . A significantly higher number of total risk alleles was found in patients with early-onset schizophrenia $(<20$ years old) than in other age groups. There was no sex-specific difference in multi-site polymorphisms among the groups. Tables 2 and 3 show MTHFR polymorphisms in patients of different age groups and total risk allele number of MTHFR in patients with schizophrenia and controls, respectively.

Table 3 Total Risk Allele Number of MTHFR in Early- and AdultOnset Schizophrenia

\begin{tabular}{|l|c|c|c|c|}
\hline MTHFR Risk Allele No. & $\mathbf{0}$ & $\mathbf{1}$ & $\mathbf{2}$ & $\mathbf{3}$ \\
\hline Early & 12 & 31 & 59 & 12 \\
Adult & 10 & 28 & 21 & 4 \\
\hline \multicolumn{4}{|c|}{$\chi^{2}=8.386^{*},{ }^{\dagger}, \mathrm{df}=3, \mathrm{p}=0.0387$} \\
\hline
\end{tabular}

Notes: ${ }^{*} p<0.05$; There was statistical difference on total risk allele number of MTHFR between early- and adult-onset groups; ${ }^{\dagger}$ Comparison in total risk allele number of MTHFR in patients with divergent onset ages.
Serum samples were used to determine folate and homocysteine levels. The results demonstrated that there was no significant difference in the levels of these two substances in patients of different ages of onset.

\section{Symptoms in Patients of Different Ages of Onset}

The analysis of the PANSS total, positive and negative scores of the patients indicated that the early-onset patients exhibited significantly higher scores relative to the results of other groups, consistent with the more severe symptoms observed in patients with early-onset schizophrenia. The subgroup analysis of both male and female patients further provided comparable results that onset in adolescents was accompanied by more severe symptoms. Figure 1 shows the PANSS scores in the early-onset group compared with the adult-onset group.

\section{MTHFR Polymorphisms and Symptoms of}

\section{Schizophrenia}

The analysis of PANSS total, positive and negative scores indicated that these scores were positively correlated with the multi-site polymorphisms of MTHFR, which only appeared in patients with early-onset schizophrenia rather than in those with later ages of onset. No sex-specific differences were observed in this result. Figure 2 shows the correlation between PANSS and MTHFR polymorphisms in the early-onset and adultonset groups.

\section{Analysis of Symptom Prediction}

Therefore, MTHFR polymorphisms have been found to be increased in patients with early-onset schizophrenia. 

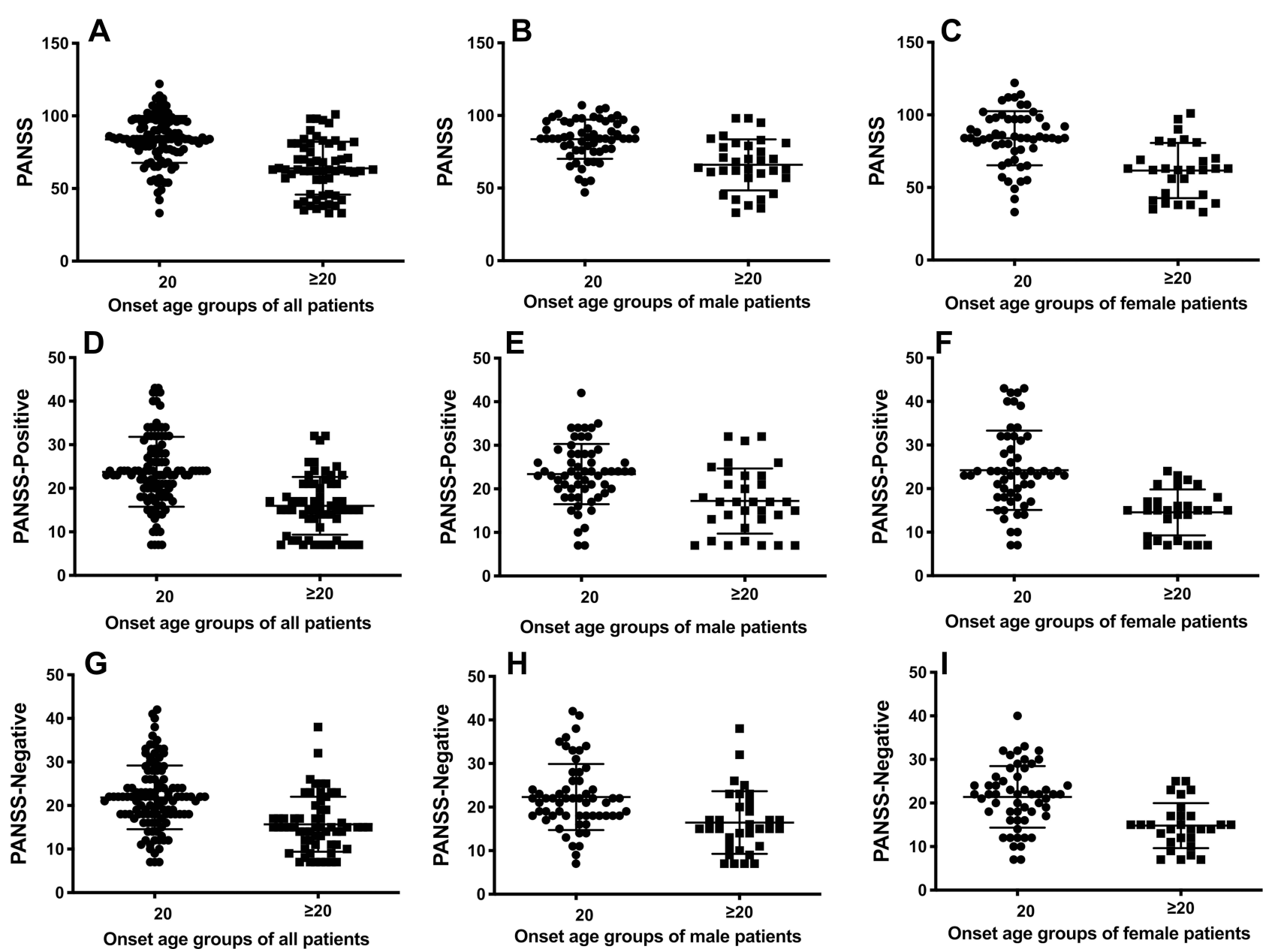

Figure I The PANSS scores of schizophrenic patients of early-onset age ( $<20$ years old) in each group were significantly higher than that of adult-onset ones $(p<0.05)$. (A) $t$

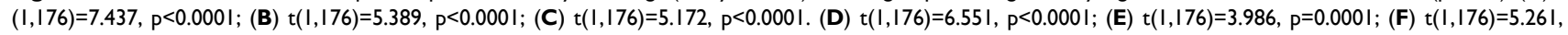
$p<0.0001 ;(\mathbf{G}) t(1,176)=5.599, p<0.0001 ;(H) t(I, 176)=3.614, p=0.0005 ;(I) t(I, I 76)=4.420, p<0.0001$.

In contrast, this group of patients' symptoms is often more severe than those of adult-onset patients. We further investigated the association between onset age and related metabolites (such as folate and Hcy) and PANSS scores of patients with schizophrenia. A multiple linear regression model was constructed to analyze their interaction by inputting onset age, folate, and Hcy levels as the independent variables and scores of symptoms as the dependent variable. As a result, earlier onset age was significantly associated with more severe symptoms, and folate levels were found to be significantly associated with PANSS total and PANSS positive in the early-onset group. Table 4 shows the correlation of multiple factors, including folate, Hcy, onset age, and PANSS. After that, the single linear regression of PANSS scores and metabolites, including folate and Hcy, showed that folate level was significantly negatively associated with PANSS and PANSS positive in the adult-onset group.

\section{Genomic Methylation Level in Different Onset Age Groups Analysis of Methylation Prediction}

As there was a significant difference in the early onset group ( $<20$ years old) compared with the others, we combined the middle- (20-30 years old) and adult-onset ( $>30$ years old) groups to perform the methylation analysis with the early onset group. Figure 3 shows the methylation levels in different onset-age groups, methylation heatmap of DMPs, and DMP enrichment genes.

There was a significant difference in DMPs in patients of different ages of onset by heatmap and ANOVA (Figure 3), which indicated that more hypomethylated positions were found in patients with early-onset than in controls. The 
A

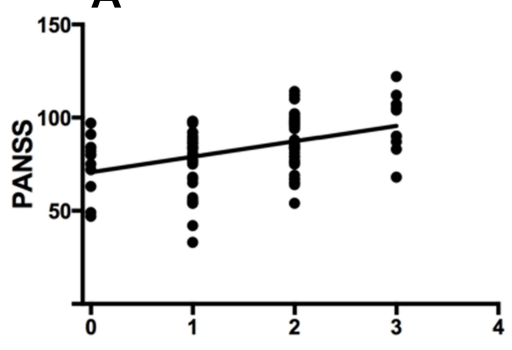

$C C=8.296 p<0.0001$

D

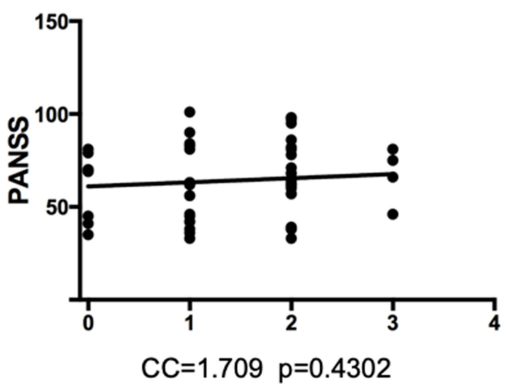

B

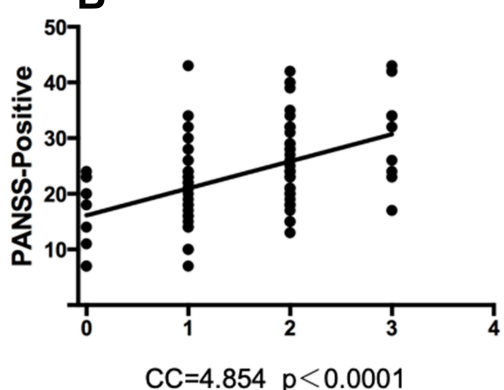

$E$

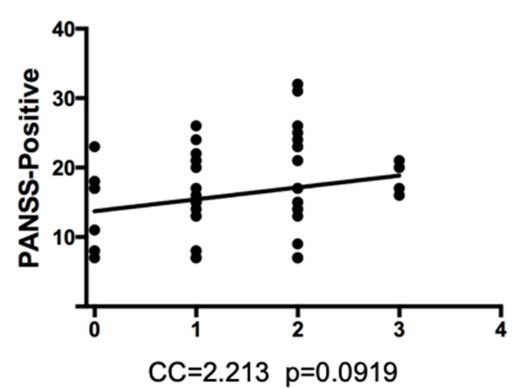

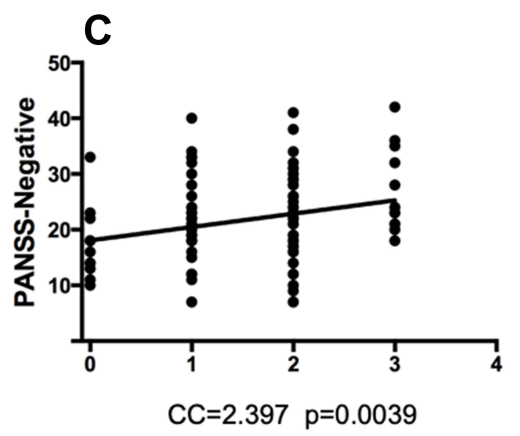

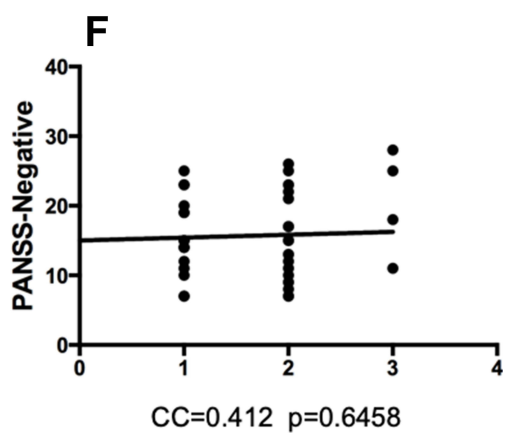

Figure 2 The PANSS scores were positively associated with the elevation of MTHFR polymorphisms in schizophrenic patients of early-onset age, but not in those of adultonset ones. Each $X$-axis means the total risk allele number of 3 MTHFR polymorphic sites (C677T, AI298C, GI793A). (A) PANSS total and MTHFR multi-site polymorphisms in patients of early-onset. $\mathrm{P}<0.000 \mathrm{I}, \mathrm{Y}=8.296 \mathrm{X}+70.7 \mathrm{I}$. (B) PANSS positive and MTHFR multi-site polymorphisms in patients of early-onset. $\mathrm{P}<0.000 \mathrm{I}$, $Y=4.854 X+16.13$. (C) PANSS negative and MTHFR multi-site polymorphisms in patients of early-onset. $p<0.01, Y=2.397 X+18.08$. (D) PANSS total and MTHFR multi-site polymorphisms in patients of adult-onset. $p>0.05, Y=1.709 X+13.73$. (E) PANSS positive and MTHFR multi-site polymorphisms in patients of adult-onset. $p>0.05, Y=2.213 X$ +6I.03. (F) PANSS negative and MTHFR multi-site polymorphisms in patients of adult-onset. $p>0.05, Y=0.4|I 9 X+| 5.02$.

genomic methylation level was also significantly different between the groups of different ages of onset. The $\beta$ values of the two groups of early- and adult-onset were $0.55 \pm 0.17$ and $0.62 \pm 0.19$ (mean $\pm \mathrm{SD}$ ), respectively (Figure 3B), indicating that there were significantly lower genomic DNA methylation levels in patients with early-onset. Subgroups of males and females also revealed a similar difference in that significant genomic hypomethylation was found in patients with early onset. Genomic methylation analysis did not reveal any gender-specific effects.

\section{Differential Methylation Positions}

The exclusion criteria were as follows: non-CpG, SNPrelated, location in the $\mathrm{X}$ or $\mathrm{Y}$ chromosome, and multi-hit. On the standard of $\Delta \beta \geq 0.10$, a total of 2,640 DMPs in patients of different age groups were identified, including 1,523 hypomethylated positions and 1,117 hypermethylated positions in early-onset subjects, respectively. The DMPs are shown in a heatmap (Figure 3A). The genes involved in DMP enrichment are shown in Figure 3C.

\section{GO Analysis}

According to the GO analysis, 600 terms containing genes with significant enrichment of DMPs were identified, including multicellular organism development and system development. The descriptive terms for the most considerable enrichment are shown in Figure 4. The figure shows the analysis screened by the classic Fisher test and 10 items with the most significance in each category of GO. Based on the previous data, the terms were characterized by containing the genes with DMP enrichment in our study and labeled them on the top of the columns.

\section{Gene-Specific DNA Methylation}

For $S L C 6 A 4$, the average methylation analysis of four $\mathrm{CpG}$ sites demonstrated that the early-onset group had hypermethylation compared with the adult-onset group. For $B D N F$, a similar analysis of six $\mathrm{CpG}$ sites found hypomethylation in the early-onset group. The quantification of COMT and NRG1 methylation did not differ between patients with early-onset and adult-onset schizophrenia. Table 5 shows the gene-specific DNA methylation levels.

\section{Discussion}

\section{Onset Age in Schizophrenia}

According to the literature, schizophrenia has been classified into different phenotypes based on the clinical features 
Table 4 Multiple Linear Regression of Folate, Hcy, Onset Age and Symptoms

\begin{tabular}{|c|c|c|c|c|}
\hline & B & Std B & SE & $95 \% \mathrm{Cl}$ \\
\hline \multicolumn{5}{|c|}{ (A) PANSS-Total } \\
\hline Folate & 1.239 & 0.180 & 0.453 & $(0.345,2.132)^{* *}$ \\
\hline Onset-age & 19.445 & 0.484 & 2.586 & $(14.340,24.550)^{* *}$ \\
\hline Interaction & -1.247 & -0.069 & 1.196 & $(-3.607,1.114)$ \\
\hline \multicolumn{5}{|c|}{ (B) PANSS-Positive } \\
\hline Folate & 0.425 & 0.142 & 0.206 & $(0.020,0.83 \mathrm{I})^{*}$ \\
\hline Onset-age & 7.642 & 0.437 & 1.175 & $(5.324,9.961)^{* *}$ \\
\hline Interaction & -0.356 & -0.045 & 0.543 & $(-1.428,0.716)$ \\
\hline \multicolumn{5}{|c|}{ (C) PANSS-Negative } \\
\hline Folate & 0.215 & 0.080 & 0.192 & $(-0.164,0.593)$ \\
\hline Onset-age & 6.073 & 0.386 & 1.095 & $(3.911,8.235)^{* *}$ \\
\hline Interaction & -0.322 & -0.045 & 0.506 & $(-1.321,0.678)$ \\
\hline \multicolumn{5}{|c|}{ (D) PANSS-Total } \\
\hline Hcy & 0.263 & 0.189 & 0.130 & $(0.005,0.520)$ \\
\hline Onset-age & 27.465 & 0.683 & 4.456 & $(|8.670,36.26|)^{* *}$ \\
\hline Interaction & -0.378 & -0.252 & 0.183 & $(-0.740,-0.016)$ \\
\hline \multicolumn{5}{|c|}{ (E) PANSS-Positive } \\
\hline Hcy & 0.133 & 0.219 & 0.058 & $(0.018,0.248)$ \\
\hline Onset-age & 11.208 & $0.64 I$ & 1.994 & $(7.273,15.144)^{* *}$ \\
\hline Interaction & -0.166 & -0.255 & 0.082 & $(-0.328,-0.005)$ \\
\hline \multicolumn{5}{|c|}{ (F) PANSS-Negative } \\
\hline Hcy & 0.093 & 0.170 & 0.054 & $(-0.014,0.200)$ \\
\hline Onset-age & 7.891 & 0.502 & 1.854 & $(4.23 \mathrm{I}, \mathrm{I} \mathrm{I} .55 \mathrm{I})^{* *}$ \\
\hline Interaction & -0.079 & -0.134 & 0.076 & $(-0.229,0.072)$ \\
\hline
\end{tabular}

Notes: ${ }^{*}<0.05 ; * * p<0.01$.

Abbreviations: B, regression coefficient; Std B, standardized $\beta$ value; SE, standard error; $95 \% \mathrm{Cl}, 95 \%$ confidence interval.

of the disease course. In addition to the complex symptoms, patients' onset age is also a notable feature in related pathogenesis studies. As mentioned previously, studies have shown that the symptoms in patients with early-onset schizophrenia are severe. ${ }^{20-22}$ To illustrate the related mechanisms of different characteristics in patients with divergent onset ages, studies on biochemical metabolites in the cortex regions have suggested that $N$-acetyl-aspartate levels are significantly lower in the left dorsolateral prefrontal cortex in patients with early-onset schizophrenia than in healthy controls, which is not observed in adult-onset patients. ${ }^{23}$
Another investigation of the onset age further suggests that D-cycloserine may aggravate the negative symptoms of early onset, supporting the biochemical basis of onset age divergence. $^{24}$ For the pathogenesis of schizophrenia, genetic factors are one of the most critical reasons that promote physiological and clinical alterations in patients with schizophrenia.

Meanwhile, no different results have been obtained from molecular biology research in patients with schizophrenia with divergent onset ages, which may significantly influence the phenotypes of schizophrenia. MTHFR is an 
A

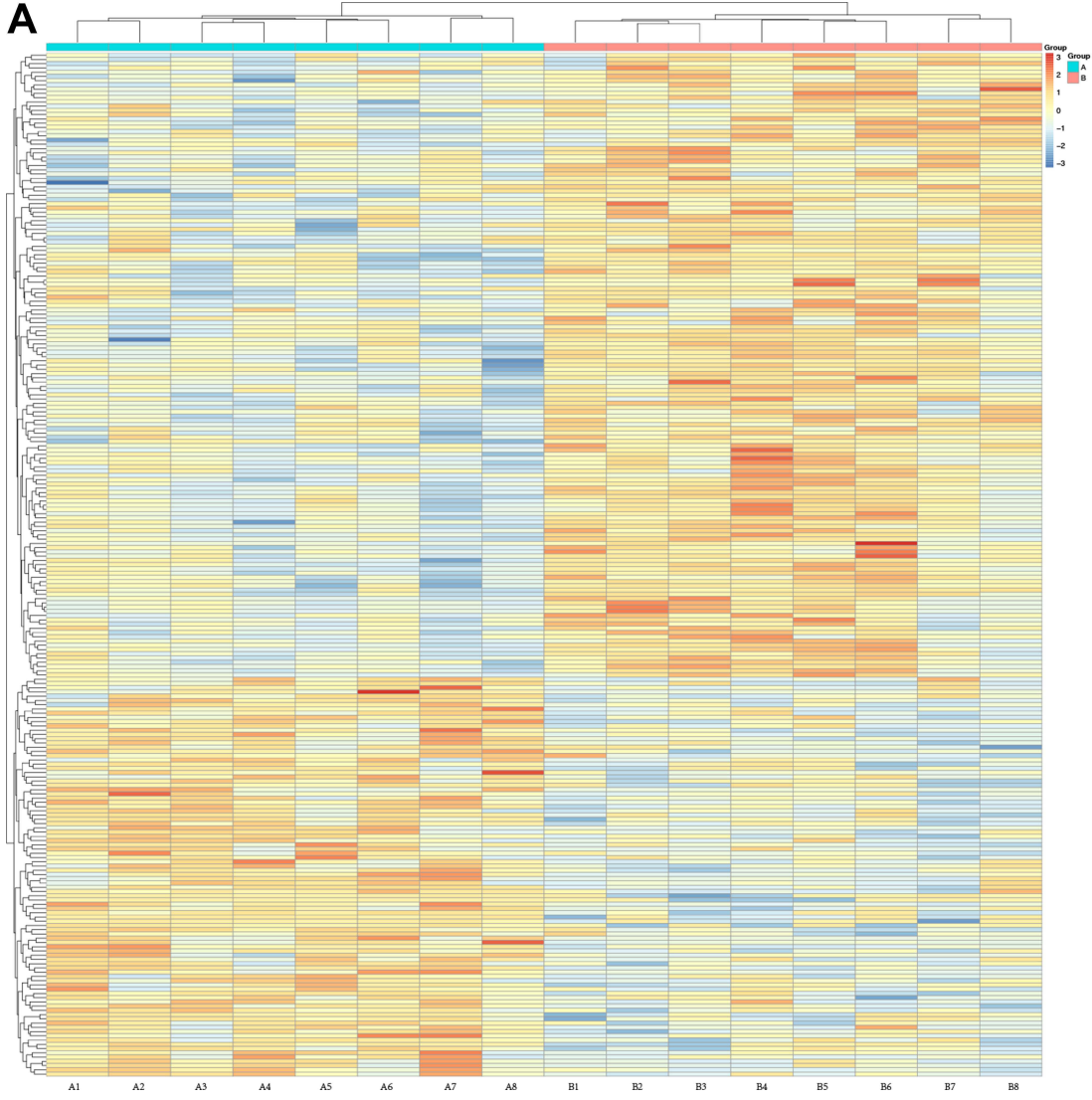

B

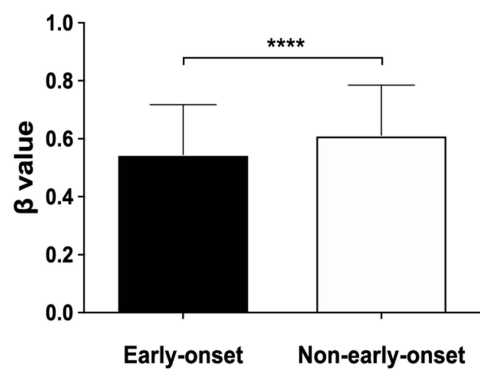

C
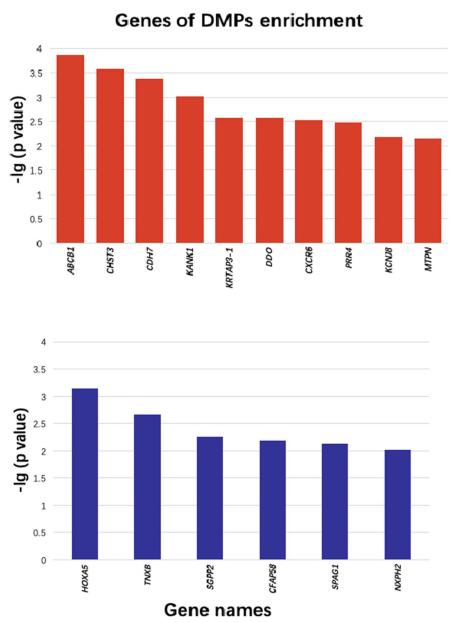

Figure 3 (A) Each box represents $\beta$ value relative to standardized conversion (z-score), integrating all $\beta$ values of samples for the one position. The mean value of each position after standardization is 0 . Each row means a position with differential methylation between schizophrenia patients of early and adult-onset, and each column means an individual enrolled. (B) The methylation level was measured in $\beta$ value. The figures show schizophrenic patients of early-onset had a hypomethylated pattern compared to others. Short bars mark the standard deviation. $F(I, 7)=4.234$, **** $p<0.000 I$. (C) Genes of DMP enrichment. Upper and lower figure shows the genes enriched by DMPs with hypo- and hypermethylation level in patients of early-onset respectively.

Abbreviations: $A B C B I$, ATP-binding cassette, subfamily B (MDR/TAP), member I; CHST3, chondroitin 6-sulfotransferase 3; CDH7, cadherin 7; KANKI, KN motif and ankyrin repeat domain-containing protein I; KRTAP3-I, keratin associated protein 3-I; DDO, D-aspartate oxidase; CXCR6, C-X-C chemokine receptor type 6; PRR4, poliovirus receptor-related 4; KCNJ8, potassium inwardly-rectifying channel subfamily J member 8; MTPN, myotrophin; HOXA5, homeobox protein Hox-A5; TNXB, tenascin XB; SGPP2, sphingosine-I-phosphate phosphatase 2; CFAP58, cilia and flagella associated protein 58; SPAGI, sperm-associated antigen I; NXPH2, neurexophilin-2.

essential enzyme that catalyzes the conversion of folate to 5-methyl folate (5-MTHF), which is correlated with the negative and cognitive symptoms of schizophrenia. ${ }^{25,26}$ The biological effects of MTHFR polymorphisms mainly lie in the enzyme activity alteration and the related downstream methyl group supply, which is critical for methylation modification. A few studies have reported a correlation between the methylation of epigenetic processes and the onset age of certain diseases, including schizophrenia. $^{14,27}$

\section{MTHFR and Onset Age in Schizophrenia}

In our study, the PANSS scores of patients with schizophrenia with different onset ages exhibited significant differences, while early-onset patients showed more severe symptoms than the other patients. This result is consistent with those of prior studies. ${ }^{28,29}$ As there is a potential association between neurodevelopment and onset age of schizophrenia, we are also interested in the developmentrelated one-carbon metabolism processes, including MTHFR polymorphisms, metabolites (such as folate or Hcy), and methylation modification downstream. Based on the correlation between MTHFR polymorphism and the divergent onset age of schizophrenia, this is the first study aiming at folate conversion-related MTHFR polymorphism and schizophrenia research grouping in divergent onset ages of patients. In contrast, we found no distinct polymorphic effect of a single site in the divergent onset age groups, while the multi-site polymorphism was significantly increased in early-onset patients. In comparison with the polymorphic effect of a single site, the polymorphic effect of multiple sites more comprehensively determines the effect of MTHFR. However, few studies on the multi-site polymorphism of MTHFR have 


\section{GO of DMP enrichment}

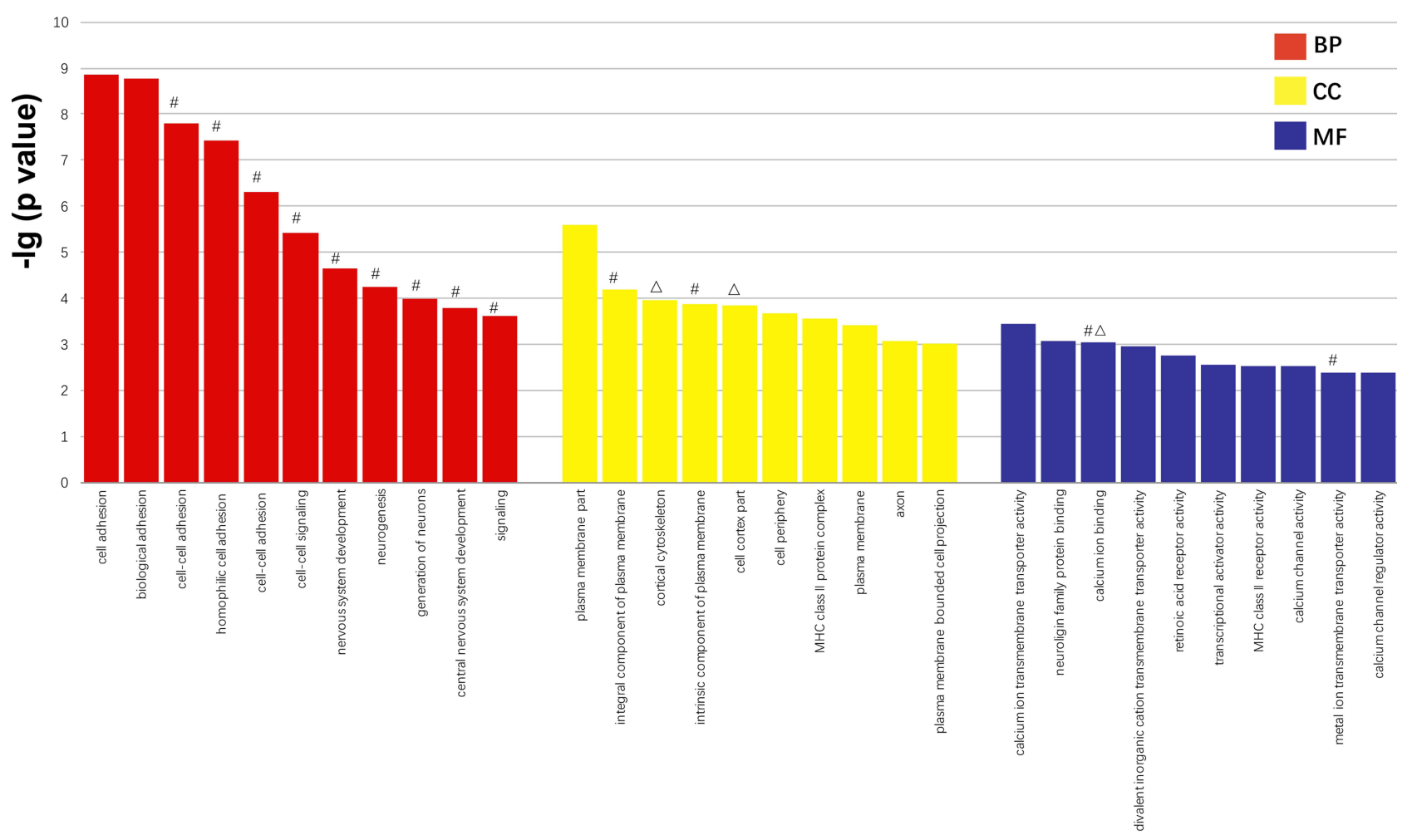

\section{GO terms}

Figure 4 Screened by classic fisher test and 10 items with the most significance in each category of GO are exhibited. The larger the vertical coordinate is, the more significant the enrichment of item is. X axis means different items with significant enrichment of GO; Yaxis means - $\log _{10}$ (adjusted $p$ value of DMPs). "The term related genes of DMP enrichment that is hypomethylated in schizophrenic patients of early-onset. ${ }^{\Delta}$ The term related genes of DMP enrichment that is hypermethylated in schizophrenic patients of early-onset.

Abbreviations: BP, biological processes; CC, cellular components; MF, molecular functions.

been published to elucidate the effects of variants, which are more relevant to enzyme activity while participating in folate conversion and directly influencing the methylation process.

Given the different degrees of symptom severity and MTHFR polymorphisms in patients with schizophrenia of divergent onset ages, we analyzed the association between MTHFR polymorphisms and PANSS scores of patients. Partly consistent with the results of related previous studies, ${ }^{18,25}$ the symptoms were all positively correlated with the MTHFR multi-site polymorphisms, which were only observed in patients with early onset ( $<20$ years old $)$ but not in patients with adult onset. The age of onset may be a factor that influences the correlation between MTHFR and symptoms. In summary, the results implied that MTHFR plays a potential role in the unique population of patients with early-onset schizophrenia. This finding is consistent with the results of previous studies that increased MTHFR polymorphism may aggravate the symptoms of schizophrenia. ${ }^{25,26}$ However, there are few studies on the correlation mentioned above in early-onset patients. The participation of MTHFR in these patients with schizophrenia implies its molecular effects in this potential special phenotype of the disease.

\section{One-Carbon Metabolites and Symptom}

Changes in molecular mechanisms, such as MTHFR polymorphism, are correlated with clinical divergence, including symptom aggravation in patients with early-onset schizophrenia. As MTHFR participates in folate metabolism, the level of the related metabolites in one-carbon metabolism (eg, folate and Hcy) has also been found to be associated with schizophrenia and symptoms. ${ }^{30-32}$ A decreased level of serum folate has been observed in patients with schizophrenia, while the degree of reduction was positive with the severity of symptoms tested using the PANSS scale. ${ }^{31}$ In 
Table 5 Gene-Specific DNA Methylation Levels

\begin{tabular}{|l|c|c|c|c|c|c|c|}
\hline \multicolumn{7}{|c|}{ (A) DNA Methylation Level of Each COMT CPG Site } \\
\hline \multicolumn{7}{|c|}{} & \multicolumn{7}{|c|}{ CPG No. } & \\
\hline CPG No. & 1 & 2 & 3 & 4 & 5 & 6 & Average \\
\hline Early & 4.86 & 0.41 & 2.49 & 1.26 & 0.64 & 3.80 & 2.24 \\
\hline Adult & 1.75 & 0.45 & 0 & 1.07 & 0.58 & 3.58 & \\
\hline
\end{tabular}

(B) DNA Methylation Level of Each SLC6A4 CPG Site

\begin{tabular}{|c|c|c|c|c|c|}
\hline \multirow[b]{2}{*}{ CpG No. } & \multicolumn{4}{|c|}{ CpG No. } & \multirow[b]{2}{*}{ Average* } \\
\hline & I & 2 & 3 & 4 & \\
\hline Early & 3.61 & 10.25 & 0.74 & 1.92 & 4.13 \\
\hline Adult & 2.04 & 8.23 & 0 & 0.92 & 2.80 \\
\hline
\end{tabular}

(C) DNA Methylation Level of Each NRGI CPG Site

\begin{tabular}{|l|c|c|c|c|c|c|c|c|}
\hline & \multicolumn{7}{|c|}{ CpG No. } & \\
\hline CpG No. & 1 & 2 & 3 & 4 & 5 & 6 & 7 & \\
\hline Early & 4.53 & 6.09 & 6.37 & 5.28 & 4.89 & 1.24 & 3.84 & Average \\
\hline Adult & 4.94 & 7.13 & 6.87 & 7.53 & 3.7 & 0.45 & 1.06 & 4.61 \\
\hline
\end{tabular}

(D) DNA Methylation Level of Each BDNF CpG Site

\begin{tabular}{|c|c|c|c|c|c|c|c|}
\hline \multirow[b]{2}{*}{ CpG No. } & \multicolumn{6}{|c|}{ CpG No. } & \multirow[b]{2}{*}{ Average* } \\
\hline & 1 & 2 & 3 & 4 & 5 & 6 & \\
\hline Early & 8.73 & 8.98 & 24.29 & 8.68 & 11.57 & 5.58 & $\mid 1.31$ \\
\hline Adult & 11.19 & 9.85 & 29.43 & 9.27 & 11.78 & 7.92 & 13.24 \\
\hline
\end{tabular}

Notes: (A) $F(I, I I)=1.94, p>0.05$; (B) $F(I, 7)=1.31$, * $p<0.05$; (C) $F(I, 13)=2.86, p>0.05$; (D) $F(I, I I)=1.47$, * $p<0.05$; Schizophrenic patients with early-onset age were hypermethylated in the analyzed region of SLC6A4 compared to adult-onset age. See Table B for the DNA methylation level of each CpG site. Data is shown by mean $\pm S E$. * $p<0.05$; Schizophrenic patients with early-onset age were hypomethylated in the analyzed region of BDNF compared to adult-onset age. See Table D for the DNA methylation level of each CPG site. Data is shown by mean \pm SE. * $p<0.05$.

addition, an elevated level of Hcy was detected in patients with schizophrenia. ${ }^{33}$ However, this conclusion cannot be reached consistently in other published studies. ${ }^{34,35}$ In this study, the levels of serum folate and Hcy in patients with schizophrenia with divergent onset ages did not show a significant difference. Folate level was positively correlated with symptom score, which is not in accordance with previous results. After the interactional analysis that entered onset-age as an independent factor, the subsequent single linear regression implies a negative correlation between folate level and symptoms. The decreased level of folate, which implies a limited trend in folate conversion and an increase in MTHFR polymorphism, may contribute to the development of schizophrenia. Owing to the global trend in the severity of symptom severity in patients with early-onset schizophrenia, the clinical phenotype alteration may be attributed to the processes related to MTHFR and folate metabolism, which participate in metabolic pathways that fluctuate in these patients.

Apart from folate and Hcy, B Vitamins including B6 and B12 were revealed effective intervention in reducing psychiatric symptoms. ${ }^{36-38}$ As metabolites in one-carbon metabolism, vitamin B6 and B12 are also important factors in schizophrenia vulnerability. Both contribute to the remission of folate deficiencies and hyperhomocysteinaemia, which are prevalent among schizophrenic patients. ${ }^{39,40}$ Then folate and homocysteine might represent the final determined roles in psychiatric disorder. The 
impact of B vitamins may involve in their changes, while could be reflected in folate and homocysteine analysis.

\section{Potential Methylation Effects}

As more specific features were found in patients with early-onset schizophrenia, more details are necessary to investigate further potential mechanisms of this phenotype in the population. In one-carbon metabolism, folate is converted to 5-MTHF, which participates in the supply of methyl groups and epigenetic modification downstream. DNA methylation frequently prevents gene expression through steric hindrance, which may be a mechanism that mediates the association between MTHFR polymorphism and schizophrenia. For DNA methylation, the levels of genomic and specific gene regions are usually measured for assessment. It has been reported that the methylation levels of genomic and specific gene regions could lead to a certain degree of alteration in patients with schizophrenia. ${ }^{14,41}$ In healthy subjects with the TT genotype of MTHFR C677T, the genomic methylation level is usually higher than that in patients with schizophrenia. ${ }^{42}$ However, only a few studies have reported a potential mechanism through methylation alteration in patients with schizophrenia of divergent onset ages. In the current study, the genomic methylation level in early-onset patients was remarkably lower than that in patients of other onset ages, and more hypomethylated positions were identified in the early-onset group. By selecting subjects whose total number of TT genotypes of MTHFR C677T and PANSS exceeded 60, we regarded the MTHFR polymorphism and clinical phenotypes of patients with schizophrenia as one unit to explore the underlying mechanisms. Subjects with these two features may reflect a more prominent alteration in the downstream methyl supply. Compared with middle/late ages of onset, in patients with early-onset schizophrenia, hypomethylation implies a potential role of methylation alteration resulting from MTHFR polymorphisms. The patients with adultonset exhibit relatively mild symptoms of the disease when severe symptomatic schizophrenia occurs in patients with homozygous mutation genotype. Genomic hypomethylation is usually associated with genomic instability. ${ }^{43}$ Some biological terms were found through methylation analysis to study the underlying mechanisms in patients with schizophrenia with divergent onset ages. More than 4,000 positions exhibited differential methylation levels among patients of different age groups, contributing to their differential genomic DNA methylation levels. We also performed GO analysis to explore the related biological processes, cellular components, and molecular functions that influence the onset of schizophrenia. We selected and labeled the GO terms containing the previous genes with DMP enrichment, which can be considered a set point for subsequent research.

Since genomic methylation may be the mechanism by which MTHFR affects the phenotype of schizophrenia with divergent ages of onset, it may be related to the specific methylation level of some disease-related genes. Serotonergic neurotransmission plays an essential role in the mechanism of action of atypical antipsychotics. Then, the regulation of serotonin transporters may be obtained by SLC6A4 epigenetic analysis. In our study, SLC6A4 in patients with early-onset schizophrenia showed significant hypermethylation, implying a potential downstream pathway of MTHFR effects through serotonin. In contrast to $S L C 6 A 4, B D N F$ indicates hypomethylation in the same group. The $B D N F$ methylation level has been shown to increase in patients with schizophrenia, ${ }^{41}$ while no research has focused on the methylation alteration of SLC6A4 or BDNF in patients with divergent onset ages. For $N R G 1$ and COMT, although there was no significant diversity in methylation levels of specific sites, the expression of related genes have been reported to associate with schizophrenia risk. ${ }^{44,45}$ Probably much more samples and research would work in the future. Therefore, genespecific methylation changes in the potential pathways of MTHFR affect the divergent onset of schizophrenia and are possible objects of research on the disease mechanism.

\section{Conclusions}

Patients with early-onset schizophrenia have more severe symptoms related to MTHFR polymorphisms and serum folate levels. In patients of this population, MTHFR polymorphisms are increased, and related genomic methylation levels are reduced, which may be the underlying mechanism of this disease course and onset age-associated phenotype. Following the biological pathways, specific genes, including SLC6A4 and BDNF, may be potential research objects for different possible phenotypes. The results would potentially imply a special phenotype of schizophrenia, which is characterized by more severe symptoms, increased MTHFR polymorphism, and decreased genomic methylation levels. The results would also facilitate research on the mechanisms and clinical practice of schizophrenia. 
While there were several points that should be clarified. Firstly, sample size for association studies (114 vs 63) were small to be analyzed, and studies with a larger sample size are required. Secondly, for MTHFR and onecarbon metabolism, it is probable that both SNP and gene expression research would work well in collaboration of fundamental research and clinical practice. Thirdly, as with short of large-sample study, subsequent studies are needed to explore their distinct effects at different ages of onset in schizophrenia and possible phenotypes by screening potential genes and pathways with enrichment of DMPs, which is more comprehensive. In future studies, we should pay attention to these issues, and an in-depth research is essential.

\section{Abbreviations}

PANSS, Positive and Negative Syndrome Scale; MTHFR, Methylenetetrahydrofolate reductase; DMPs, Differential methylation positions; COMT, CatecholO-methyltransferase; SLC6A4, Solute carrier family 6 member 4; NRG1, Neuregulin 1; BDNF, Brain-derived neurotrophic factor; DSM-5, Diagnostic and Statistical Manual of Mental Disorders, Fifth Edition; Hcy, Homocysteine.

\section{Data Sharing Statement}

Data collected for this study will not be made available to others.

\section{Ethics Approval and Informed Consent}

This study protocol and informed consent forms were approved by the Ethics committee of Peking Union Medical College Hospital.

\section{Consent for Publication}

No patient data are identifiable, thus no consent is required.

\section{Acknowledgments}

We thank for all patients for their participation in the study, and Drs. and clinical researchers from Peking Union Medical College Hospital and Beijing Anding Hospital for their support and help. We are thankful to Peking Union Medical College Hospital, Beijing Anding Hospital for providing access to experiments and technical support.

\section{Author Contributions}

All authors made substantial contributions to conception and design, acquisition of data, or analysis and interpretation of data; took part in drafting the article or revising it critically for important intellectual content; agreed to submit to the current journal; gave final approval of the version to be published; and agree to be accountable for all aspects of the work.

\section{Funding}

1) National Key R\&D Program of China, No. 2016YFC1307205. 2) Peking Union Medical College Hospital Funding Project of Chinese Academy of Medical Sciences, grant number zc201902261.

\section{Disclosure}

The authors report no conflicts of interest for this work and declare that the research was conducted in the absence of any commercial or financial relationships that could be construed as a potential conflict of interest.

\section{References}

1. Carlsson A, Carlsson ML. A dopaminergic deficit hypothesis of schizophrenia: the path to discovery. Dialogues Clin Neurosci. 2006;8(1):137-142.

2. An der Heiden W, Hafner H. The epidemiology of onset and course of schizophrenia. Eur Arch Psychiatry Clin Neurosci. 2000;250 (6):292-303. doi:10.1007/s004060070004

3. Nicolson R, Brookner FB, Lenane M, et al. Parental schizophrenia spectrum disorders in childhood-onset and adult-onset schizophrenia. Am J Psychiatry. 2003;160(3):490-495. doi:10.1176/appi.ajp.160.3.490

4. Gogtay N, Giedd JN, Lusk L, et al. Dynamic mapping of human cortical development during childhood through early adulthood. Proc Natl Acad Sci USA. 2004;101(21):8174-8179. doi:10.1073/ pnas. 0402680101

5. Mehler C, Warnke A. Structural brain abnormalities specific to childhood-onset schizophrenia identified by neuroimaging techniques. J Neural Transm. 2002;109(2):219-234. doi:10.1007/ s007020200019

6. Cornblatt BA, Lencz T, Smith CW, Correll CU, Auther AM, Nakayama E. The schizophrenia prodrome revisited: a neurodevelopmental perspective. Schizophr Bull. 2003;29 (4):633-651. doi:10.1093/oxfordjournals.schbul.a007036

7. Clemmensen L, Vernal DL, Steinhausen HC. A systematic review of the long-term outcome of early onset schizophrenia. BMC Psychiatry. 2012;12:150. doi:10.1186/1471-244X-12-150

8. Kezurer N, Galron D, Golan HM. Increased susceptibility to mild neonatal stress in MTHFR deficient mice. Behav Brain Res. 2013;253:240-252. doi:10.1016/j.bbr.2013.07.037

9. Muntjewerff JW, Hoogendoorn ML, Kahn RS, et al. Hyperhomocysteinemia, methylenetetrahydrofolate reductase 677TT genotype, and the risk for schizophrenia: a Dutch population based case-control study. Am J Med Genet B Neuropsychiatr Genet. 2005;135B(1):69-72. doi:10.1002/ajmg.b.30179

10. Kempisty B, Mostowska A, Gorska I, et al. Association of $677 \mathrm{C}>\mathrm{T}$ polymorphism of methylenetetrahydrofolate reductase (MTHFR) gene with bipolar disorder and schizophrenia. Neurosci Lett. 2006;400(3):267-271. doi:10.1016/j.neulet.2006.02.055 
11. Vares M, Saetre P, Deng H, et al. Association between methylenetetrahydrofolate reductase (MTHFR) C677T polymorphism and age of onset in schizophrenia. Am J Med Genet B. 2010;153b(2):610-618. doi:10.1002/ajmg.b.31030

12. Muntjewerff JW, Ophoff RA, Buizer-Voskamp JE, Strengman E, den Heijer M, Consortium G. Effects of season of birth and a common MTHFR gene variant on the risk of schizophrenia. Eur Neuropsychopharmacol. 2011;21(4):300-305. doi:10.1016/j. euroneuro.2010.10.001

13. Saetre P, Vares M, Werge T, et al. Methylenetetrahydrofolate reductase (MTHFR) C677T and A1298C polymorphisms and age of onset in schizophrenia: a combined analysis of independent samples. $A m$ $J$ Med Genet B Neuropsychiatr Genet. 2011;156(2):215-224. doi:10.1002/ajmg.b.31160

14. Melas PA, Rogdaki M, Osby U, Schalling M, Lavebratt C, Ekstrom TJ. Epigenetic aberrations in leukocytes of patients with schizophrenia: association of global DNA methylation with antipsychotic drug treatment and disease onset. FASEB J. 2012;26 (6):2712-2718. doi:10.1096/fj.11-202069

15. Frosst P, Blom HJ, Milos R, et al. A candidate genetic risk factor for vascular disease: a common mutation in methylenetetrahydrofolate reductase. Nat Genet. 1995;10(1):111-113. doi:10.1038/ng0595-111

16. van der Put NM, Gabreels F, Stevens EM, et al. A second common mutation in the methylenetetrahydrofolate reductase gene: an additional risk factor for neural-tube defects? Am J Hum Genet. 1998;62 (5):1044-1051. doi:10.1086/301825

17. Goff DC, Bottiglieri T, Arning E, et al. Folate, homocysteine, and negative symptoms in schizophrenia. Am J Psychiatry. 2004;161 (9):1705-1708. doi:10.1176/appi.ajp.161.9.1705

18. Roffman JL, Brohawn DG, Nitenson AZ, Macklin EA, Smoller JW, Goff DC. Genetic variation throughout the folate metabolic pathway influences negative symptom severity in schizophrenia. Schizophr Bull. 2013;39(2):330-338. doi:10.1093/schbul/sbr150

19. Wan L, Zhang G, Liu M, Wang C, Li Y, Li R. Sex-specific effects of methylenetetrahydrofolate reductase polymorphisms on schizophrenia with methylation changes. Compr Psychiatry. 2019;94:152121. doi:10.1016/j.comppsych.2019.152121

20. Alaghband-Rad J, McKenna K, Gordon CT, et al. Childhood-onset schizophrenia: the severity of premorbid course. J Am Acad Child Adolesc Psychiatry. 1995;34(10):1273-1283. doi:10.1097/00004583199510000-00012

21. Asarnow JR, Tompson MC, McGrath EP. Annotation: childhood-onset schizophrenia: clinical and treatment issues. $J$ Child Psychol Psychiatry. 2004;45(2):180-194. doi:10.1111/ j.1469-7610.2004.00213.x

22. Badura F, Trott GE, Mehler-Wex C, et al. A study of cranial computer tomograms in very early and early onset schizophrenia. J Neural Transm. 2001;108(11):1335-1344. doi:10.1007/s007020100010

23. Stanley JA, Vemulapalli M, Nutche J, et al. Reduced N-acetylaspartate levels in schizophrenia patients with a younger onset age: a single-voxel 1H spectroscopy study. Schizophr Res. 2007;93(13):23-32. doi:10.1016/j.schres.2007.03.028

24. Takiguchi K, Uezato A, Itasaka M, et al. Association of schizophrenia onset age and white matter integrity with treatment effect of D-cycloserine: a randomized placebo-controlled double-blind crossover study. BMC Psychiatry. 2017;17(1):249. doi:10.1186/s12888017-1410-3

25. Roffman JL, Weiss AP, Purcell S, et al. Contribution of methylenetetrahydrofolate reductase (MTHFR) polymorphisms to negative symptoms in schizophrenia. Biol Psychiatry. 2008;63(1):42-48. doi:10.1016/j.biopsych.2006.12.017

26. Roffman JL, Weiss AP, Deckersbach T, et al. Effects of the methylenetetrahydrofolate reductase (MTHFR) C677T polymorphism on executive function in schizophrenia. Schizophr Res. 2007;92(13):181-188. doi:10.1016/j.schres.2007.01.003
27. Gijselinck I, Van Mossevelde S, van der Zee J, et al. The C9orf72 repeat size correlates with onset age of disease, DNA methylation and transcriptional downregulation of the promoter. Mol Psychiatry. 2016;21(8):1112-1124. doi:10.1038/mp.2015.159

28. Bettes BA, Walker E. Positive and negative symptoms in psychotic and other psychiatrically disturbed children. J Child Psychol Psychiatry. 1987;28(4):555-568. doi:10.1111/j.1469-7610.1987. tb00223.x

29. DeLisi LE. The significance of age of onset for schizophrenia. Schizophr Bull. 1992;18(2):209-215. doi:10.1093/schbul/18.2.209

30. Cao B, Wang DF, Xu MY, et al. Lower folate levels in schizophrenia: a meta-analysis. Psychiatry Res. 2016;245:1-7. doi:10.1016/j. psychres.2016.03.003

31. Song X, Fan X, Li X, et al. Serum levels of BDNF, folate and homocysteine: in relation to hippocampal volume and psychopathology in drug naive, first episode schizophrenia. Schizophr Res. 2014;159(1):51-55. doi:10.1016/j.schres.2014.07.033

32. Kinoshita M, Numata S, Tajima A, et al. Cumulative effect of the plasma total homocysteine-related genetic variants on schizophrenia risk. Psychiatry Res. 2016;246:833-837. doi:10.1016/j. psychres.2016.10.017

33. Geller V, Friger M, Sela BA, Levine J. Elevated homocysteine level in siblings of patients with schizophrenia. Psychiatry Res. 2013;210 (3):769-772. doi:10.1016/j.psychres.2013.08.016

34. Petronijevic ND, Radonjic NV, Ivkovic MD, et al. Plasma homocysteine levels in young male patients in the exacerbation and remission phase of schizophrenia. Prog Neuropsychopharmacol Biol Psychiatry. 2008;32(8):1921-1926. doi:10.1016/j.pnpbp.2008.09.009

35. Bromberg A, Levine J, Nemetz B, Belmaker RH, Agam G. No association between global leukocyte DNA methylation and homocysteine levels in schizophrenia patients. Schizophr Res. 2008;101(13):50-57. doi:10.1016/j.schres.2008.01.009

36. Lerner V, Miodownik C, Kaptsan A, Cohen H, Loewenthal U, Kotler M. Vitamin B6 as add-on treatment in chronic schizophrenic and schizoaffective patients: a double-blind, placebo-controlled study. $J$ Clin Psychiatry. 2002;63(1):54-58. doi:10.4088/jcp. v63n0111

37. Hill M, Shannahan K, Jasinski S, et al. Folate supplementation in schizophrenia: a possible role for MTHFR genotype. Schizophr Res. 2011;127(1-3):41-45. doi:10.1016/j.schres.2010.12.006

38. Roffman JL, Lamberti JS, Achtyes E, et al. Randomized multicenter investigation of folate plus vitamin B12 supplementation in schizophrenia. JAMA Psychiatry. 2013;70(5):481-489. doi:10.1001/ jamapsychiatry.2013.900

39. Misiak B, Frydecka D, Slezak R, Piotrowski P, Kiejna A. Elevated homocysteine level in first-episode schizophrenia patients-the relevance of family history of schizophrenia and lifetime diagnosis of cannabis abuse. Metab Brain Dis. 2014;29(3):661-670. doi:10.1007/ s11011-014-9534-3

40. Moustafa AA, Hewedi DH, Eissa AM, Frydecka D, Misiak B. Homocysteine levels in schizophrenia and affective disorders-focus on cognition. Front Behav Neurosci. 2014;8:343. doi:10.3389/ fnbeh.2014.00343

41. Teroganova N, Girshkin L, Suter CM, Green MJ. DNA methylation in peripheral tissue of schizophrenia and bipolar disorder: a systematic review. BMC Genet. 2016;17:27. doi:10.1186/s12863016-0332-2

42. Friso S, Choi SW, Girelli D, et al. A common mutation in the 5,10-methylenetetrahydrofolate reductase gene affects genomic DNA methylation through an interaction with folate status. Proc Natl Acad Sci U S A. 2002;99(8):5606-5611. doi:10.1073/ pnas.062066299

43. Eden A, Gaudet F, Waghmare A, Jaenisch R. Chromosomal instability and tumors promoted by DNA hypomethylation. Science. 2003;300(5618):455. doi:10.1126/science.1083557 
44. Bray NJ, Buckland PR, Williams NM, et al. A haplotype implicated in schizophrenia susceptibility is associated with reduced COMT expression in human brain. Am J Hum Genet. 2003;73(1):152-161. doi: $10.1086 / 376578$
45. Munafo MR, Thiselton DL, Clark TG, Flint J. Association of the NRG1 gene and schizophrenia: a meta-analysis. Mol Psychiatry. 2006;11(6):539-546. doi:10.1038/sj.mp.4001817

\section{Publish your work in this journal}

Neuropsychiatric Disease and Treatment is an international, peerreviewed journal of clinical therapeutics and pharmacology focusing on concise rapid reporting of clinical or pre-clinical studies on a range of neuropsychiatric and neurological disorders. This journal is indexed on PubMed Central, the 'PsycINFO' database and CAS, and is the official journal of The International Neuropsychiatric Association (INA). The manuscript management system is completely online and includes a very quick and fair peer-review system, which is all easy to use. Visit http://www.dovepress.com/testimonials.php to read real quotes from published authors.

Submit your manuscript here: https://www.dovepress.com/neuropsychiatric-disease-and-treatment-journal 